\title{
Mid ventricular obstruction in hypertrophic obstructive cardiomyopathy New diagnostic and therapeutic challenge
}

\author{
RAUL E. FALICOV AND LEON RESNEKOV \\ From the Section of Cardiology, Department of Medicine, The University of Chicago, Chicago, \\ Illinois, U.S.A.
}

A recent editorial by Shah (1975) outlined the eventful and controversy-filled saga of hypertrophic obstructive cardiomyopathy (HOCM) (idiopathic hypertrophic subaortic stenosis, muscular subaortic stenosis, obstructive asymmetric septal hypertrophy). Concepts of the anatomical and pathophysiological mechanism(s) of left ventricular outflow obstruction present in this disease have evolved slowly over the years. At first, obstruction was attributed to a circular muscle band in the left ventricular outflow tract (Brachfeld and Gorlin, 1959; Brock, 1959; Cohen et al., 1964) but later this view was felt to be too simple, overlooking the fact that the left ventricle unlike the right does not have a true muscular outflow tract (Shah, 1975). Whether a true intraventricular pressure gradient was actually even present in hypertrophic cardiomyopathy was subsequently questioned by Criley et al. (1965). They explained recorded subaortic pressure gradients as being caused by entrapment of the catheter, based on the demonstration that high subendocardial pressures transmitted to a catheter embedded in the trabeculated left ventricular apex result in artefactual gradients (Morrow et al., 1965; Dieudonné, 1966). Further consideration of the problem indicated that systolic outflow obstruction does occur in hypertrophic cardiomyopathy, coexisting in some instances with artefactual gradients caused by catheter entrapment (Adelman and Wigle, 1968). The criteria for discrimination between true and artefactual intraventricular gradients were described by Ross et al. (1966) and by Wigle et al. (1967) and were based mainly on recording a high left ventricular inflow systolic pressure (obtained by withdrawal of a transseptal catheter from the left ventricle to the left atrium) as the primary evidence for true obstruction. Angiographic observations had already suggested that the anterior leaflet of the mitral valve could be an important cause of ventricular obstruction by its abnormal apposition to the septum during systole. Outflow obstruction in hypertrophic cardiomyopathy was, therefore, defined by recording a systolic pressure gradient between the inflow and outflow portions of the left ventricle; the finding of high apical pressures in the presence of low left ventricular inflow pressures was then to be interpreted as catheter entrapment artefact resulting from the vigorous ventricular emptying during systole.

More recently, echocardiographic observations have provided additional information regarding the possible causes of systolic outflow obstruction in hypertrophic obstructive cardiomyopathy. The septum is disproportionately thicker than the posterior wall by a ratio of 1.3 or more (Henry et al., 1973a) and the anterior mitral leaflet moves in systole toward the septum (Shah et al., 1969). The length and degree of the systolic apposition of the mitral leaflet against the septum have correlated well with outflow gradients found at catheterisation (Henry et al., 1973b), though lack of correlation has been noted by other authors (Rossen et al., 1974). The precise mechanisms for the systolic apposition of the anterior mitral leaflet against the septum are not clearly understood, being attributed to malalignment of the anterior papillary muscle (Reis et $a l ., 1974$ ) or to a Venturi effect on the left ventricular outflow tract (Wigle et al., 1971) produced by the abnormal anatomy and contraction patterns. As further evidence of the role of the mitral valve, Cooley et al. (1973) showed disappearance of outflow gradients after mitral valve replacement with a 
low profile prosthesis in several patients. Both echocardiographic and haemodynamic observations, then, appeared closely correlated and seemed to have shown, with reasonable certainty, the precise site and structures involved in producing left ventricular outflow obstruction in hypertrophic obstructive cardiomyopathy.

Recent findings, however, suggest that the pathophysiological and anatomical alterations in hypertrophic obstructive cardiomyopathy may be wider ranging than previously thought. In a recent, comprehensive review of 40 postmortem hearts of patients who had hypertrophic obstructive cardiomyopathy, Roberts and Ferrans (1975) found that ventricular septal thickness was not greater than free wall thickness in 7 hearts $(18 \%)$ negating in these the presence of asymmetric septal hypertrophy in the anatomical sense. Septal thickness was also greatest in all of their cases midway between the aortic valve cusps and the left ventricular apex, rather than in the outflow tract itself. In 11 hearts $(28 \%)$ there was no thickening of the mural endocardium in apposition to the anterior mitral leaflet, suggesting that systolic mitral valve apposition to the septum had not been a prominent feature during life. Thirtythree patients had had cardiac catheterisation studies, and a subaortic gradient was shown in 20 , but there is no mention by the authors of the route of left ventricular catheterisation, or whether left ventricular inflow tract pressures were measured.

We have recently reported 2 patients (Falicov et al., 1976) in whom left ventricular obstruction was present in the mid ventricular area, rather than at the junction of the inflow and outflow tracts. In the first patient, the diagnosis was made by postmortem examination after sudden death with unrelieved obstruction 6 months after septal myotomymyectomy of the left ventricular outflow tract by the transaortic approach. In the second patient, a twocatheter left ventricular study (transseptal and retrograde) showed no gradient between the inflow and outflow tracts, but a high, true pressure gradient between the apex and the inflow and outflow tracts; the gradient was present at a sphincter-like severe narrowing in the mid ventricular area. In retrospect, identical angiographic features were present in the first patient. The second patient's echocardiogram showed asymmetric septal hypertrophy but no systolic anterior motion of the mitral leaflet in spite of a resting $100 \mathrm{mmHg}$ intraventricular systolic gradient. We suggested that obstruction in these patients was caused by a true muscular stenosis in the mid portion of the left ventricle. Since the abnormality was in the mid portion of the ventricle and the anterior leaflet of the mitral valve was not participating in the obstruction, neither outflow septal myectomy nor mitral valve removal alone could reasonably be expected to relieve the transventricular pressure gradient. On the other hand, mid ventricular septal resection and/or papillary muscle and trabecular resection might well relieve the obstruction by removing some of the hypertrophic muscular mass in the mid ventricular zone, the cause of the obstruction.

We have more recently had the opportunity of testing this hypothesis (Falicov et al., 1977) in a patient with severe mid ventricular obstruction (resting systolic gradient of $75 \mathrm{mmHg}$ ), shown by the two-catheter technique mentioned above, and additional mitral valve stenosis as well (mitral valve area $=1.3 \mathrm{~cm}^{2}$, thought to be rheumatic in origin. Echocardiogram showed severe, asymmetric hypertrophy of the posterior wall in the mid ventricular area and no systolic anterior movement of the anterior mitral leaflet. At operation, the mitral valve was resected and replaced with a Hancock porcine heterograft valve. The papillary muscles were excised as close to their bases as possible, and several hypertrophic trabeculations were resected in the mid ventricular area from the free wall as well as the septum. At postoperative catheterisation, the mid ventricular systolic gradient was reduced to one-half of its preoperative value, and the transmitral diastolic gradient was abolished.

There is, therefore, now sufficient evidence accumulated to support the concept of a spectrum of physiopathological and anatomical disorders causing left ventricular obstruction in hypertrophic obstructive cardiomyopathy, which probably relate to the heterogeneous and disorderly hypertrophic process underlying this disease. In many cases, the abnormal systolic contraction patterns and deformed geometry of the left ventricular cavity alter the direction of papillary muscle contraction and produce haemodynamic forces which attract the anterior mitral valve leaflet against the bulging septum during systole; thus, obstruction occurs at the junction of the inflow and outflow tracts. In other instances, the hypertrophic process predominates at the mid ventricular level, and the hypertrophic papillary muscles together with abnormal free wall trabeculations abut against the septum during systole to produce true muscular stenosis; there is a high pressure apical chamber, while both inflow and outflow tracts remain free of obstruction (Fig. 1). Obstruction may even be present in varying degrees at both outflow and mid ventricular levels as reported by E. D. Wigle (1976, personal communication) and may be explained by a combination of some or all the factors already mentioned. 


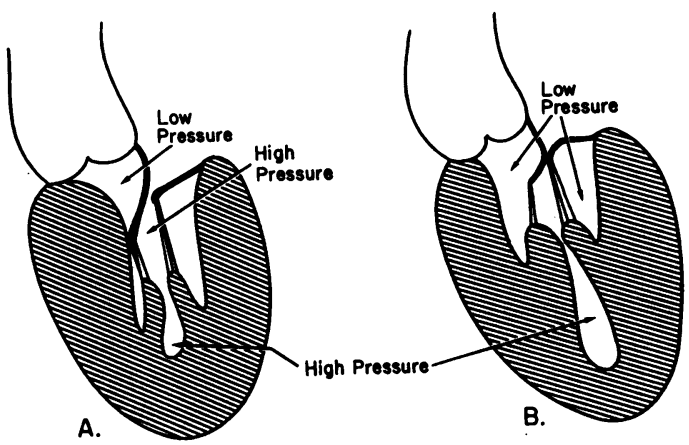

Fig. 1 Schematic representation of the two possible sites of systolic obstruction in hypertrophic obstructive cardiomyopathy. (A) Subaortic obstruction, produced by systolic apposition of the mitral leaflet against the septum. (B) Mid ventricular obstruction, produced by muscular apposition in the mid ventricular area.

\section{DIAGNOSIS OF MID VENTRICULAR HYPERTROPHIC OBSTRUCTIVE CARDIOMYOPATHY}

The clinical presentation of our patients was indistinguishable from that of other patients with subaortic hypertrophic obstructive cardiomyopathy in terms of symptoms, clinical signs, and electrocardiographic features. The echocardiogram in two patients showed some suggestive features, such as the absence of true systolic anterior motion of the anterior leaflet of the mitral valve in both patients, and lack of asymmetrical septal hypertrophy in one (in fact, she had asymmetrical hypertrophy of the posterior wall). Thus, the presence of atypical echocardiographic features in patients with otherwise typical hypertrophic obstructive cardiomyopathy may alert the clinician to the possible presence
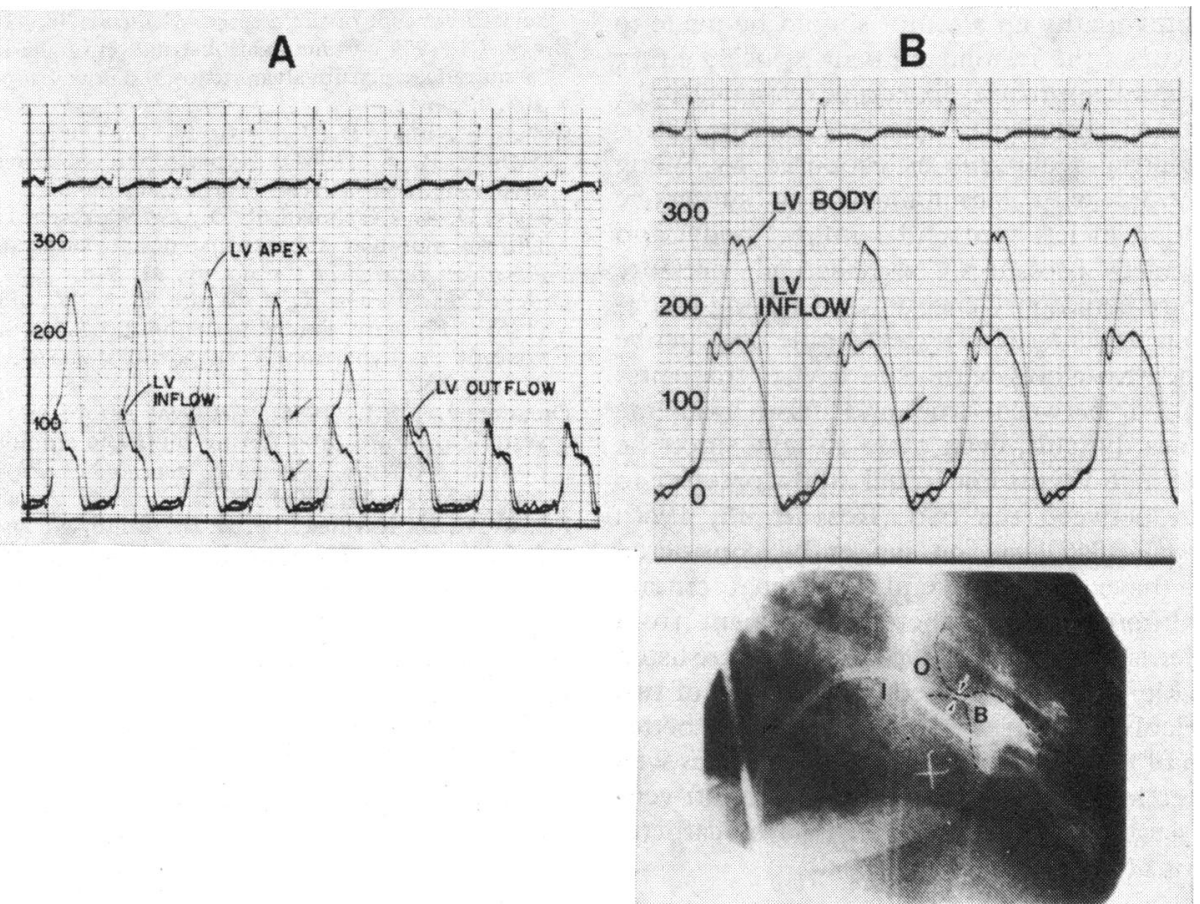

Fig. 2 Two types of gradients in a patient with mid ventricular hypertrophic obstructive cardiomyopathy.

(A) $A$ transseptal catheter was recording left ventricular inflow tract pressures ( $L V$ inflow). A second catheter with multiple side holes had been advanced retrogradely into the left ventricular (LV) body and was pulled back into the outflow tract ( $L V$ outflow). Notice the delayed fall in LV pressure (arrows) while high pressure was being recorded, suggesting catheter entrapment. Blood could still be withdrawn from the catheter during systole, indicating that some of the side holes were free in the $L V$ cavity, while others were trapped against the $L V$ wall and recording subendocardial pressure. (B) In the same patient, a single, end-hold catheter was placed during a second study in the left ventricular cavity while a transseptal catheter was placed in the $L V$ inflow tract. The fall in $L V$ systolic pressure in both tracings occurred at the same time (arrow), blood could be withdrawn from the catheter during systole, and manual injection of contrast (bottom) outlined a blood-filled cavity surrounding the tip of the catheter, with a severe muscular narrowing immediately downstream from it (arrows). A true transventricular gradient was therefore demonstrated. $I=$ inflow tract, $O=$ outflow tract, $B=$ left ventricular body. 
of mid ventricular hypertrophic obstructive cardiomyopathy.

Until more experience is accumulated, however, the mainstay for the diagnosis of mid ventricular hypertrophic obstructive cardiomyopathy rests on the typical angiographic features ('hour-glass' ventricle), together with the precise localisation of a true gradient in the mid left ventricular area. An informal survey of major cardiac catheterisation facilities in the Chicago metropolitan area, with a combined study load of over 2800 patients annually, showed that 1 per cent of patients studied over the past year had hypertrophic obstructive cardiomyopathy. Most were investigated using an aortic or brachial arterial catheter and a second catheter placed in the left ventricle by the retrograde approach; thus, no effort was made to locate the level of the gradient. We believe that in every patient studied because of suspected hypertrophic obstructive cardiomyopathy an attempt should be made to locate the site of the systolic gradient by using either the transseptal approach, in conjunction with the retrograde left ventricular approach, or the retrograde left atrial technique as proposed by Wong et al. (1975), with simultaneous or successive determination of left ventricular inflow, body, and outflow tract pressures. Artefactual pressure gradients are commonly encountered during catheterisation in patients with hypertrophic obstructive cardiomyopathy along with true cavity gradients; differentiation between true and false gradients is sometimes difficult, even more so now since the criterion of high versus low initial inflow pressure to distinguish between the two (Ross et al., 1966; Wigle et al., 1967) can no longer be considered valid. We have found several additional criteria useful in differentiating catheter entrapment from true gradients (Wigle et al., 1967) and they are listed in the Table. The simultaneous recording of two intraventricular pressures is recommended for better evaluation of the magnitude of the gradients as well as the detection of the delayed fall in the left ventricular systolic pressure characteristic of catheter entrapment artefact (Fig. 2).

Table Recognition of catheter entrapment: catheter entrapment artefacts are common in hypertrophic obstructive cardiomyopathy and coexist with true gradients

Use catheter with end hole, no side holes

Unusual waveform

Longer duration of systole (delayed relaxation)

Inability to aspirate blood during systole

Intramyocardial staining by hand injection of contrast during systole

(Modified from Wigle et al., 1967)
It is clear that more than a casual approach is needed for the detection of mid ventricular obstructive cardiomyopathy, but we believe that an effort in this direction is definitely worth while. Besides the serious practical implications regarding the surgical approach to this disease as documented in two of our patients, the determination of the actual prevalence of mid ventricular obstructive cardiomyopathy will help stimulate further thinking about the pathophysiology, diagnosis, and management of hypertrophic obstructive cardiomyopathy, a 'disease of pitfalls and paradoxes' (Spodick, 1972).

\section{References}

Adelman, A. G., and Wigle, E. D. (1968). Two types of intraventricular pressure difference in the same patient. Circulation, 38, 649-655.

Brachfeld, N., and Gorlin, R. (1959). Subaortic stenosis: a revised concept of the disease. Medicine, 38, 415-433.

Brock, R. (1959). Functional obstruction of the left ventricle (acquired aortic subvalvar stenosis). Guy's Hospital Reports, 108, 126-143.

Cohen, J., Effat, H., Goodwin, J. F., Oakley, C. M., and Steiner, R. E. (1964). Hypertrophic obstructive cardiomyopathy. British Heart fournal, 26, 16-32.

Cooley, D. A., Leachman, R. D., and Wukasch, D. C. (1973). Diffuse muscular subaortic stenosis: surgical treatment. American fournal of Cardiology, 31, 1-6.

Criley, J. M., Lewis, K. B., White, R. I., Jr., and Ross, R. S. (1965). Pressure gradients without obstruction. A new concept of 'hypertrophic subaortic stenosis'. Circulation, 32, 881-887.

Dieudonné, J. M. (1966). Artificial nature and site of the systolic pressure drop during inotropic stimulation of the normal ventricle. Canadian fournal of Physiology and Pharmacology, 44, 829-836.

Falicov, R. E., Karunaratne, H. B., Cahill, N, and Lamberti, J. J., Jr. (1977). Hypertrophic cardiomyopathy with midventricular obstruction associated with mitral stenosis: partial relief of the obstruction by papillary muscle and trabecular resection. Fournal of Catheterization and Cardiovascular Diagnosis (in press).

Falicov, R. E., Resnekov, L. Bharati, S., and Lev, M. (1976). Mid-ventricular obstruction: a variant of obstructive cardiomyopathy. American fournal of Cardiology, 37, 432.

Henry, W. L., Clark, C. E., and Epstein, S. E. (1973a). Asymmetric septal hypertrophy (ASH): the unifying link in the IHSS disease spectrum. Circulation, 47, 827-832.

Henry, W. L., Clark, C. E., Glancy, D. L., and Epstein, S. E. (1973b). Echocardiographic measurement of the left ventricular outflow gradient in idiopathic hypertrophic subaortic stenosis. New England fournal of Medicine, 288, 989-993.

Morrow, A. G., Vasko, J. S., Henney, R. P., and Brawley, R. K. (1965). Can outflow obstruction be induced within the normal left ventricle? American Fournal of Cardiology, 16, 540-546.

Reis, R. L., Bolton, M. R., King, J. F., Pugh, D. M., Dunn, M. I., and Mason, D. T. (1974). Anterior-superior displacement of papillary muscles producing obstruction and mitral regurgitation in idiopathic hypertrophic subaortic stenosis. Circulation, 49-50, Suppl. II, II-181-188.

Roberts, W. C., and Ferrans, V. J. (1975). Pathologic anatomy of the cardiomyopathies. Human Pathology, 6, 287-342. 
Ross, J., Jr., Braunwald, E., Gault, J. H., Mason, D. T., and Morrow, A. G. (1966) The mechanism of the intraventricular pressure gradient in idiopathic hypertrophic subaortic stenosis. Circulation, 34, 558-578.

Rossen, R. M, Goodman D. J., Ingham, R. E., and Popp, R. L. (1974). Echocardiographic criteria in the diagnosis of idiopathic hypertrophic subaortic stenosis. Circulation, 50, 747-751.

Shah, P. (1975). IHSS-HOCM-MSS-ASH? Circulation, 51, 577-580.

Shah, P. M., Gramiak, R., and Kramer, D. H. (1969). Ultrasound localization of left ventricular outflow obstruction in hypertrophic obstructive cardiomyopathy. Circulation, 40, 3-11.

Spodick, D. H. (1972). Hypertrophic obstructive cardiomyopathy of the left ventricle. Cardiovascular Clinics, 4 (1), p. 133. F. A. Davis, Philadelphia.

Wigle, E. D., Adelman, A. G., and Silver, M. D. (1971).
Pathophysiological considerations in muscular subaortic stenosis. In Hypertrophic Obstructive Cardiomyopathy, p. 63. (Ciba Foundation Study Group No. 37.) Ed. by G. E. W. Wolstenholme and M. O'Connor Churchill, London.

Wigle, E. D., Marquis, Y., and Auger, P. (1967). Muscular subaortic stenosis. Initial left ventricular inflow tract pressure in the assessment of intraventricular pressure differences in man. Circulation, 35, 1100-1117.

Wong, B., Langou, R. A., Cohen, L. S., and Wolfson (1975). Retrograde catheterization of the left atrium in idiopathic hypertrophic subaortic stenosis. American fournal of Cardiology, 36, 62-66.

Requests for reprints to Dr. Raul E. Falicov, Section of Cardiology, Christ Hospital, 4440 W. 95th Street, Oak Lawn, Illinois 60453, U.S.A. 